\title{
Mobile Monitoring of Diabetic Patients in the Active File of the Medical Center of Do (Burkina Faso)
}

\author{
Bry SYLLA ${ }^{a}$, Seydou Golo BARRO ${ }^{\mathrm{b}, 1}$, Jeoffray DIENDERE ${ }^{\mathrm{c}}$, Rachid G. KONFE ${ }^{\mathrm{a}}$, \\ Ghislain BOUDA ${ }^{\mathrm{d}}$ and Pascal STACCINI ${ }^{\mathrm{e}}$ \\ ${ }^{a}$ Health Information System Department, Ministry of Health, Burkina Faso \\ ${ }^{\mathrm{b}}$ Nazi BONI University, Bobo-Dioulasso, Burkina Faso \\ ${ }^{\mathrm{c}}$ Health Sciences Research Institute (IRSS) \\ ${ }^{\mathrm{d}}$ Health District of Do, Burkina Faso \\ e RETINES Lab - Cote-d'Azur University, Nice, France
}

\begin{abstract}
The care of diabetic patients in peripheral medical centers in Burkina Faso faces many difficulties. This work, which is a new experience, aimed to set up an information system for the care of diabetic patients in the context of Burkina Faso. The system thus conceived consists of a web application (MedshakeEHR), used by the doctor and a mobile application (Glucosio) for the patient. The system has advantages such as remote appointment scheduling, appointment reminder, patient information sharing. The device also makes it possible to store data for the production of statistics and for scientific research. This experience has enabled us to meet certain challenges related in particular to the problem of HIS such as organizational constraints, the creation of a unique identifier, the modeling of the main business processes, etc.
\end{abstract}

Keywords: HIS; ICT and health; Diabetes; Telemedicine

\section{Introduction}

Burkina Faso, like most African countries, is facing an epidemiological transition in health with the significant increase in chronic non-communicable diseases. These are cardiovascular diseases, diabetes, cancers requiring regular treatment throughout the patient's life. According to WHO, the number of people with diabetes worldwide increased from 108 million in 1980 to 422 million in 2014. In the African region the prevalence fell from $3.1 \%$ to $7.1 \%$ over the same period. New eating habits, sedentary lifestyle or even genetic predispositions are the major causes of the spread of this disease [1]. Unfortunately, our health systems have not adapted to these phenomena at the same speed, which naturally has consequences on the quality of life of patients and those around them. The solution to these difficulties lies in setting up an information system to effectively monitor patients and build a database for statistics and research on diabetes. The search for electronic experiments in this area and in our context has remained poor.

This is why we have undertaken to design and implement an electronic register of diabetic patients at the Medical Center with Surgical Antenna (CMA) of the health dis-

\footnotetext{
1 Corresponding Author, Seydou Golo Baroo, University of Nazi BONI, Bobo-Dioulasso, Burkina Faso; E-mail: seydou.golo@gmail.com.
} 
trict of DO, Bobo-Dioulasso, Burkina Faso. It is an application that integrates an appointment reminder system and patient access to their data via a mobile phone system. The use of telehealth has shown potential for the management of chronic pathologies and research [2]. The interest of our system is to improve the follow-up of diabetic patients with a view to their empowerment but also to contribute to improving the quality of data on this pathology.

\section{Methods}

\subsection{System implementation site}

The implementation site of our system is the Medical Center with Surgical Antenna (CMA) of the Do health district in Bobo Dioulasso (Burkian Faso). The health district of Do has, in addition to the CMA, twenty-seven (27) peripheral Health Centers.

\subsection{Modeling and choice of tools}

The analysis of the existing system and the new system to be implemented was carried out using UML (Unified Modeling Language). The offline version of draw.io and the power AMC software were used for the diagrams. Our system is an application made up of two parts: a web part used by doctors and a mobile part used by the patient. Opensource software called "MedshakeEHR" was used to design the web application. MedshakeEHR is a Hospital Information System (HIS). The mobile part was developed using Android software which is also open source "Glucosio". For our work, we used the Apache HTTP server version 2.4.3 which is free software respecting the client-server communication protocol. From this server, our application runs through a browser, regardless of the power of the computer or the operating system used. We preferred the Linux 18.04.2 operating system for this work. and the "MySQL" Database Management System. Mobile app users were equipped with a smartphone with Android OS version 4 and later operating system. To establish the connection between MedshakeEHR and Glucosio and facilitate data exchange, we have set up an API (Application Programming Interface). We choose the "REST" API (REpresentational State Transfer) because it meets the norms and standards in data exchange. This API retrieves data from MedshakeEHR and provides it to Glucosio in JSON (JavaScript Object Notation) format. For the safety and reliability of exchanges, we have integrated a unique patient identification system using the method proposed by the GMSIH (Group for the Modernization of the Hospital Information System). This method recommends the use of identity traits to generate the unique identifier by an automatic algorithmic calculation process; this process was used by ASIP Santé during its national health identification program [3] [4].

\section{Results}

\subsection{System analysis and modeling}

The analysis of the new system defines the role of each actor as well as the data provided and consulted. For the doctor, he connects to the "MedshakeEHR" application for recording the data of the patient's medical consultation; As for the patient, he uses the "Glucosio" mobile phone part to make his appointments and consult his personal information [figure 1]. 
To record a consultation, the doctor logs into the MedshakeEHR application after mandatory authentication. He then accesses the MedshakeEHR home interface and can finally record a medical consultation. The "appointment making" process involves the patient, the doctor and the two applications. The patient uses the mobile application for the appointment request which sends a notification to the web application that will be processed by the doctor.

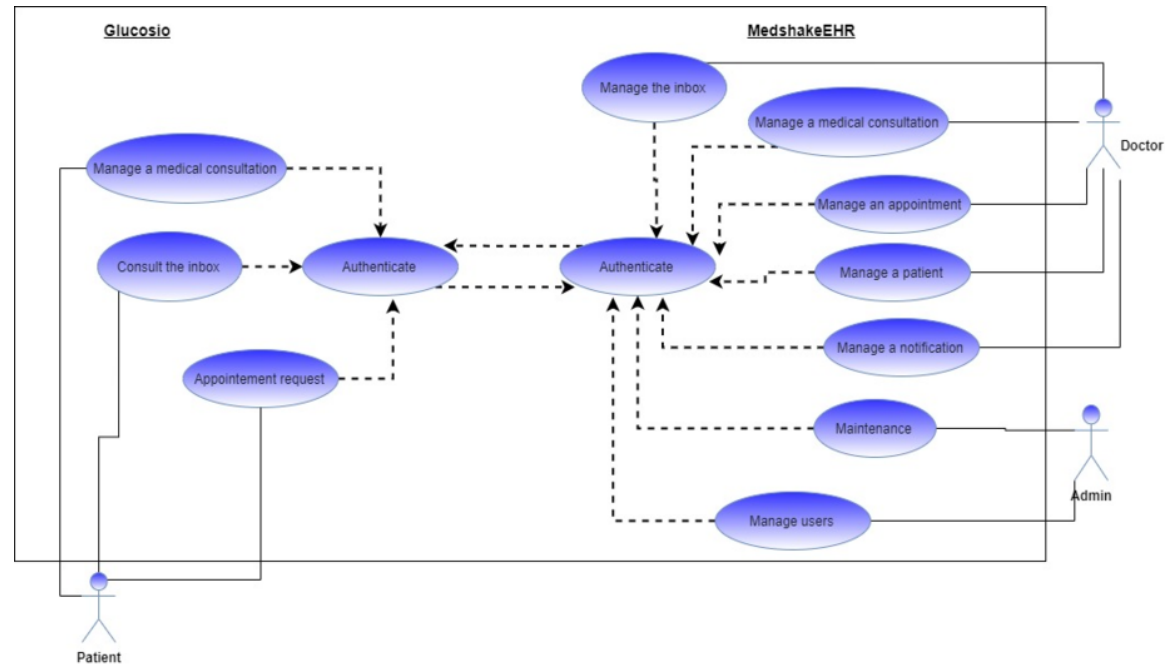

Figure 1. Use case Diagram

\subsection{System implementation}

The installation of the application was done on a desktop computer in the doctor's consulting room. Two user accounts have been created for the two physicians responsible for consulting diabetic patients with different levels of access. Security measures have been taken: an automatic backup system on external media, user authentication, limitation of user access rights according to their profile, etc. Physical measures were also taken regarding access to the consultation room and the technical room. For the protection of personal data, legal measures relating to digital health data, governed by Article 15 of Law 010-2004 / AN on the protection of personal data have been taken [5]. A system of data quality controls has been put in place during and after data entry. An interface for managing appointments and notifications has been created. For the use of the mobile phone part of the "Glucosio" application, a user account has been created allowing the patient to authenticate himself before accessing the content of the application. For security reasons, patient access has been limited to some information. The "REST" API, which was deployed on the same server as MedshakeEHR, allows the two applications to communicate with each other and facilitate the exchange of information between two or more doctors, but also between doctors and patients. When Glucosio is first installed, the patient's unique identifier will be stored on the application database. When requesting Glucosio, the unique identifier is used by the API to query MedshakeEHR and authorize secure exchanges [figure 2].

The system thus put in place has been operational since May 6, 2021. All users have been trained. We administered a system evaluation questionnaire to physicians responsible for 
consulting diabetic patients with respect to the ergonomics of the application, functionality and handling. Another interview guide was used to assess patient satisfaction. The results of this assessment show that the application is generally satisfactory (70\%). At the time of writing this article 36 patients were already registered in the system.

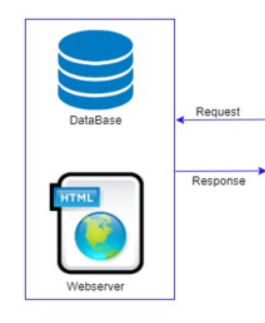

MedshakeEHR

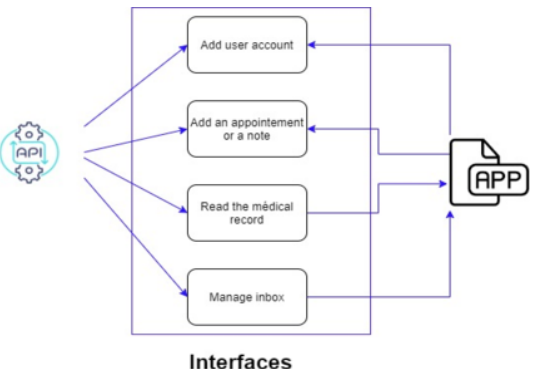

Interfaces

Figure 2. Application interface architecture

\section{Conclusions}

The electronic monitoring system for diabetic patients set up at the Do Medical Center is an innovation in the management of chronic diseases in the healthcare system of the country. It is a system with a dual objective (clinical and research) and a dual advantage (for the doctor and for the patient). Nevertheless, our project has its limits, in particular its restriction to a single medical center and a single pathology, the lack of perspective for a good evaluation, especially the clinical research component, etc. However, these limits remain as challenges to be taken up for future digital health projects in the care of patients. We set ourselves as a perspective the gradual integration of different pathologies to make this system a complete computerized patient record; the development of a billing module; the use of other features of MedshakeEHR in particular: Apycript mail, HPRIM, DICOM. It is also planned to install the system in the endocrinology department of the Souro Sanou University Hospital Center (CHUSS). Enabling the sending of SMS in addition to emails and notifications will greatly improve the management of appointment reminders in view of the mobile network coverage much higher than that of the Internet.

\section{References}

[1] Organisation Mondiale de la Santé (OMS). Rapport mondial sur le diabète. http://www.who.int/diabetes/global-report/fr/.

[2] Guo X, Gu X, Jiang J, Li H, Duan R, Zhang Y, Sun L, Bao Z, Shen J, Chen F. A HospitalCommunity-Family-Based Telehealth Program for Patients With Chronic Heart Failure: Single-Arm, Prospective Feasibility Study. JMIR Mhealth Uhealth. 2019 Dec 13;7(12):e13229. doi: $10.2196 / 13229$.

[3] ANAP. Identification du patient. https://www.anap.fr/ressources/publications/detail/actualites/identification-du-patient/.

[4] Programme Identifiant National calculé (INS-C) de Santé, Algorithme de calcul. ASIP Santé/ PRAS ed. Paris: ASIP Santé, 2009;5-9. Accessible à: (https://esante.gouv.fr/securite/identifiant-national-de-sante/ins-c).

[5] CIL. Législation nationale. http://www.cil.bf/index.php/legislation/legislation-nationale. Date d'accès : 20/05/2019. 